\title{
Editorial
}

\section{Be in Control before You Lose Control}

Thomas Gregor Issac ${ }^{1}$

\author{
${ }^{1}$ Geriatric Psychiatry Unit, Department of Psychiatry, National \\ Institute of Mental Health and Neurosciences Bangalore, \\ Karnataka, India
}

J Neurosci Rural Pract:2020;11:365-365

Suicide is an important cause for death across the globe and the World Health Organization denotes it as the second largest contributor in mortality in between 15 and 29 years of age as well as a global phenomenon of which more than three quarters $(76 \%)$ is contributed by the developing and low-income countries, but the risk factors which predispose to the same, especially in the context of mood disorders have not been studied in great detail. ${ }^{1,2}$

This study by Subramanian et al (2020) titled "study of risk factors associated with suicide attempt in patients with bipolar I disorder" tries to look into the various risk factors which are associated with suicide attempt in patients with bipolar I disorder. ${ }^{3}$ It has tried to comprehensively look into the role of sociodemographic characteristics, the clinical course of bipolar illness and association with type of polarity of mood episodes, stressful life events, coping skills, and personality factors.

Almost equivalent number of male and female patients were recruited and more than a third (36.3\%) had a history of deliberate self-harm which points to an increased vulnerability of this population for likely suicide attempt when compared with the risk in general population which is approximately $1 \%$ as per the National Mental Health Survey (2016). ${ }^{2}$

The study also reports of the various risk factors like a positive family history as an important risk factor for suicide in this specific population of patients with bipolar illness. The odds ratio of 13.65 (95\% confidence interval: $1.28-145.38, p=$ 0.030 ) itself in this study is an important eye-opener. However, it is in tune with the previous literature which also emphasizes role of previous suicidal attempts as an important risk factor. ${ }^{3-5}$ Also, it should be noted that compared with general population, patients with bipolar illness are also vulnerable to life stressors and adverse life events making them more vulnerable for suicide. Index episode of depression that has been elicited in this study as an important risk factor also needs to be understood in perspective of implication in management. Treatment of bipolar depression aggressively (especially when there is clinical suspicion of bipolar illness in background of an index depressive episode) in developing countries therefore assumes importance. Special populations like patients in postpartum depression and those with substance abuse need aggressive management. ${ }^{7}$ Difficulty in problem-focused disengagement is another important parameter which potentiates risk for suicide as described in this study. Behavioral disengagement is often considered to be an important coping mechanism which can help to protect from suicides. Hence, this study is in liaison with previous studies which also points to the need for problem-focused disengagement as an important factor negotiating likelihood for suicidal risk., ${ }^{5,7}$

The study could have fared better by incorporating factors of mode of suicide, the intentionality and lethality, awareness regarding the recent legislative amendment for decriminalization of suicide as well as the various roles of substance use and other drugs which are important in patients with bipolar illness and can predispose to suicides. It is prudent to note that many more of such studies are required in larger numbers to understand the various risk factors involved in suicide. Primordial prevention in terms of recognition and mitigation of these risk factors have a long way in prevention of suicide as social isolation has in prevention of coronavirus disease 2019 infection.

\section{Conflict of Interest}

None declared.

\section{References}

1 Ahmed HU, Hossain MD, Aftab A, et al. Suicide and depression in the World Health Organization south-east asia region: a systematic review. WHO South-East Asia J Public Health 2017;6(1):60-66

2 Pradeep BS, Gururaj G, Varghese M, et al. National mental health survey of India, 2016 - rationale, design and methods. PLOS ONE 2018;13(10):e0205096

3 Subramanian K, Menon V, Sarkar S, Chandrasekaran V, Selvakumar N. Study of risk factors associated with suicide attempt in patients with bipolar disorder - type I. J Neurosci Rural Pract 2020; 11(02): 291-298

4 Hansson C, Joas E, Pålsson E, Hawton K, Runeson B, Landén M. Risk factors for suicide in bipolar disorder: a cohort study of 12 850 patients. Acta Psychiatr Scand 2018;138(5):456-463

5 Bobo WV, Na PJ, Geske JR, McElroy SL, Frye MA, Biernacka JM. The relative influence of individual risk factors for attempted suicide in patients with bipolar I versus bipolar II disorder. J Affect Disord 2018;225:489-494

6 Coryell W, Kriener A, Butcher B, et al. Risk factors for suicide in bipolar I disorder in two prospectively studied cohorts. J Affect Disord 2016;190:1-5

7 Horwitz AG, Hill RM, King CA. Specific coping behaviors in relation to adolescent depression and suicidal ideation. J Adolesc 2011;34(5):1077-1085
Address for correspondence

Thomas Gregor Issac, Geriatric Psychiatry Unit, Department of Psychiatry,

National Institute of Mental Health and

Neurosciences, Bangalore, Karnataka, India

(e-mail: thomasgregorissac@gmail.com).
DoI https://doi.org/

10.1055/s-0040-1713343 ISSN 0976-3147.
(C)2020 Association for Helping Neurosurgical Sick People
License terms

() (1) $\ominus \circledast$ 